\title{
A unifying geometric representation for central projection systems
}

\author{
João P. Barreto \\ Institute for Systems and Robotics, Department of Electrical and Computer Engineering, University of Coimbra, 3030 Coimbra, Portugal \\ Received 7 July 2005; accepted 7 June 2006 \\ Available online 25 July 2006 \\ Communicated by Seth Teller
}

\begin{abstract}
In this paper, we study projection systems with a single effective viewpoint, including combinations of mirrors and lenses (catadioptric) as well as just lenses with or without radial distortion (dioptric systems). First, we extend a well-known unifying model for central catadioptric systems to incorporate a class of dioptric systems with radial distortion. Second, we provide a new representation for the image plane of central systems. This representation is the lifting through a Veronese map of the original image plane to the 5D projective space. We study how a collineation in the original image plane can be transferred to a collineation in the lifted space, and we prove that in the case of central parabolic systems and cameras with lens distortion the locus of the lifted points representing projections of world lines is a plane. The similarities between paracatadioptric systems and lens with radial distortion are emphasized by extending to the latter algorithms initially established for the former.
\end{abstract}

(C) 2006 Elsevier Inc. All rights reserved.

Keywords: Catadioptrics; Radial distortion; Lifting of coordinates; Veronese maps

\section{Introduction}

A vision system has a single viewpoint whenever it measures the intensity of light traveling along rays which intersect in a single point in 3D (the projection center). Vision systems satisfying the single viewpoint constraint are called central projection systems. The perspective camera is an example of a central projection system. In this case the mapping in homogeneous coordinates of points in the scene into points in the image is linear and can be described by a $3 \times 4$ projection matrix $\mathbf{P}$ (pin-hole model) [1]. Perspective projection can be modeled by intersecting a plane with a pencil of lines going through the scene points and the projection center $\mathbf{O}$.

There are central projection systems whose geometry can not be described using the conventional pin-hole model. In [2] Baker et al. derive the entire class of catadioptric systems satisfying the single viewpoint constraint. Sensors

E-mail address: jpbar@deec.uc.pt.

$U R L$ : www.deec.uc.pt/ jpbar. with a wide field of view and a unique projection center can be built by combining a hyperbolic mirror with a perspective camera, or a parabolic mirror with an orthographic camera (paracatadioptric system). However, the mapping between points in the 3D world and points in the image is non-linear. Svoboda et al. show that in general the central catadioptric image of a line is a conic section [3]. A unifying theory for central catadioptric systems is proposed in [4]. Geyer et al. prove that central catadioptric projection is isomorphic to a projective mapping from a sphere, centered in the effective viewpoint, to a plane with the projection center on the perpendicular to the plane. Perspective cameras with non-linear lens distortion are another example of central projection systems where the relation in homogeneous coordinates between scene points and image points is no longer linear. True lens distortion curves are typically very complex and higher-order models are introduced to approximate the distortion during calibration $[5,6]$. However, simpler low-order models can be used for many computer vision applications where an accuracy in the order of a pixel is sufficient. In this paper the 
radial lens distortion is modeled after the division model proposed in $[7,8]$. The division model is not an approximation to the classical model in [5], but a different approximation to the true curve.

The present work proposes, a general image formation model that accommodates conventional perspective cameras, central catadioptric systems, and dioptric cameras with lens distortion. The model is linearized by lifting through Veronese maps the projective plane in the fivedimensional space. Such an embedding provides new insights about the geometric properties of each type of projection. The contributions can be summarized as follows:

(1) The unifying model of central catadioptric systems proposed in [4] is extended to include radial distortions. It is proved that the division model for lens distortion is equivalent to a projective mapping from a paraboloid to a plane, orthogonal to the paraboloid's axis, and with projection center in the vertex of the paraboloid. It is also shown that in general the distorted projection of a line is a conic curve.

(2) For both catadioptric and radially distorted dioptric systems, we establish a new representation through lifting of the image plane to a five-dimensional projective space. In this lifted space, a collineation in the original plane corresponds to a collineation of the lifted points. We know that world lines project to conic sections whose representatives in the lifted space lie on a quadric. We prove that in the cases of parabolic catadioptric projection and radial lens distortion this quadric degenerates to a hyperplane.

\section{A unifying model for perspective cameras, central catadioptric systems, and lenses with radial distortion}

In [4], a unifying model for all central catadioptric systems is proposed where conventional perspective imaging appears as a particular case. This section reviews this image formation model as well as the result that in general the catadioptric image of a line is a conic section [3]. This framework can be easily extended to cameras with radial distortion where the division model $[7,8]$ is used to describe the lens distortion.

This section shows that conventional perspective cameras, central catadioptric systems, and cameras with radial distortion underly one projection model. Fig. 1 is a scheme of the proposed unifying model. A point in the scene $\mathbf{X}$ is transformed into a point $\mathbf{x}$ by a conventional projection matrix $\mathbf{P}$. Vector $\mathbf{x}$ can be interpreted both as a $2 \mathrm{D}$ point expressed in homogeneous coordinates, and as a projective ray defined by points $\mathbf{X}$ and $\mathbf{O}$ (the projection center). Function $\boldsymbol{\hbar}$ transforms $\mathbf{x}$ in the intermediate point $\mathbf{x}^{\prime}$. Point $\mathbf{x}^{\prime}$ is related with the final image point $\mathbf{x}^{\prime \prime}$ by function $ð$. Both $\boldsymbol{h}$ and $\circlearrowright$ are transformations defined in the two dimensional oriented projective space [9]. They can be linear or non-linear depending on the type of system, but they are always injective functions with an inverse. Table 1 summarizes the results derived along this section.

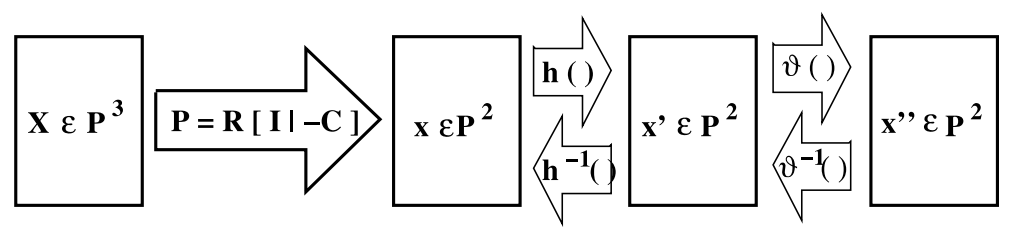

Fig. 1. Steps of the unifying model. The 3D point $\mathbf{X}$ is projected into point $\mathbf{x}=\mathbf{P X}$ assuming the conventional pin-hole model. To each point $\mathbf{x}$ corresponds an intermediate point $\mathbf{x}^{\prime}$ which is mapped in the final image plane by function $ð$. Depending on the sensor type, functions $\boldsymbol{\hbar}$ and $ð$ can represent a linear transformation or a non-linear mapping (see Table 1).

Table 1

The mapping functions $\boldsymbol{\hbar}$ and $ð$ and corresponding inverses

\begin{tabular}{|c|c|}
\hline $\begin{array}{l}\text { Perspective camera }(\xi=0, \psi=0) \\
\quad \boldsymbol{\hbar}(\mathbf{x})=(x, y, z)^{\mathrm{T}} \\
\quad \boldsymbol{\hbar}^{-1}\left(\mathbf{x}^{\prime}\right)=\left(x^{\prime}, y^{\prime}, z^{\prime}\right)^{\mathrm{T}}\end{array}$ & $\begin{array}{l}ð\left(\mathbf{x}^{\prime}\right)=\mathbf{K} \mathbf{x}^{\prime} \\
\partial^{-1}\left(\mathbf{x}^{\prime \prime}\right)=\mathbf{K}^{-1} \mathbf{x}^{\prime \prime}\end{array}$ \\
\hline 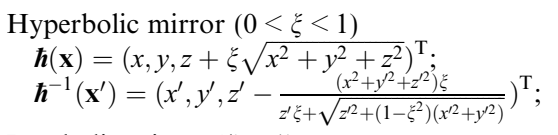 & $\begin{array}{l}ð\left(\mathbf{x}^{\prime}\right)=\mathbf{H}_{\mathbf{c}} \mathbf{x}^{\prime} \\
\partial^{-1}\left(\mathbf{x}^{\prime \prime}\right)=\mathbf{H}_{\mathbf{c}}^{-1} \mathbf{x}^{\prime \prime}\end{array}$ \\
\hline $\begin{array}{l}\text { Parabolic mirror }(\xi=1) \\
\quad \boldsymbol{h}(\mathbf{x})=\left(x, y, z+\sqrt{x^{2}+y^{2}+z^{2}}\right)^{\mathrm{T}} \\
\quad \boldsymbol{\hbar}^{-1}\left(\mathbf{x}^{\prime}\right)=\left(2 x^{\prime} z^{\prime}, 2 y^{\prime} z^{\prime}, z^{\prime 2}-x^{\prime 2}-y^{\prime 2}\right)^{\mathrm{T}}\end{array}$ & $\begin{array}{l}ð\left(\mathbf{x}^{\prime}\right)=\mathbf{H}_{\mathbf{c}} \mathbf{x}^{\prime} \\
ð^{-1}\left(\mathbf{x}^{\prime \prime}\right)=\mathbf{H}_{\mathbf{c}}^{-1} \mathbf{x}^{\prime \prime}\end{array}$ \\
\hline $\begin{array}{l}\text { Radial distortion }(\xi<0) \\
\quad \succsim\left(\mathbf{x}^{\prime}\right)=\left(2 x^{\prime}, 2 y^{\prime}, z^{\prime}+\sqrt{z^{\prime 2}-4 \xi\left(x^{\prime 2}+y^{\prime 2}\right)}\right)^{\mathrm{T}} \\
\quad \partial^{-1}\left(\mathbf{x}^{\prime \prime}\right)=\left(x^{\prime \prime} z^{\prime \prime}, y^{\prime \prime} z^{\prime \prime}, z^{\prime 2}+\xi\left(x^{\prime \prime 2}+y^{\prime \prime 2}\right)\right)^{\mathrm{T}}\end{array}$ & $\begin{array}{l}\boldsymbol{\hbar}(\mathbf{x})=\mathbf{K} \mathbf{x} \\
\boldsymbol{h}^{-1}\left(\mathbf{x}^{\prime}\right)=\mathbf{K}^{-1} \mathbf{x}^{\prime} ;\end{array}$ \\
\hline
\end{tabular}




\subsection{Perspective camera and central catadioptric systems}

The image formation in central catadioptric systems can be split in three steps [10] as shown in Fig. 1: world points are mapped into an oriented projective plane by a conventional $3 \times 4$ projection matrix $\mathbf{P}$; the oriented projective plane is transformed by a non-linear function $\boldsymbol{\hbar}$ (Eq. (1)); the last step is a collineation in the plane $\mathbf{H}_{\mathbf{c}}$ (Eq. (2)). In this case, the function $\partial$ is a linear transformation depending on the camera intrinsics $\mathbf{K}_{\mathbf{c}}$, the relative rotation between the camera and the mirror $\mathbf{R}_{\mathbf{c}}$, and the shape of the reflective surface. As discussed in $[4,10]$, parameters $\xi$ and $\psi$ in Eqs. 1 and 2 , depend only on the system type and shape of the mirror. For paracatadioptric systems $\xi=1$, while in the case of conventional perspective cameras $\xi=0$. If the mirror is hyperbolic then $\xi$ takes values in the range $[0,1]$.

$$
\begin{aligned}
\mathbf{x}^{\prime}= & \boldsymbol{h}(\mathbf{x})=\left(\boldsymbol{x}, \boldsymbol{y}, \boldsymbol{z}+\xi \sqrt{\boldsymbol{x}^{2}+\boldsymbol{y}^{2}+\boldsymbol{z}^{2}}\right)^{\mathrm{T}} \\
\mathbf{x}^{\prime \prime}= & \underbrace{\mathbf{K} \mathbf{R}_{\mathbf{c}}\left[\begin{array}{ccc}
\psi-\xi & 0 & 0 \\
0 & \xi-\psi & 0 \\
0 & 0 & 1
\end{array}\right]}_{\mathbf{H}_{\mathbf{c}}} \boldsymbol{\hbar}(\mathbf{x})
\end{aligned}
$$

The non-linear characteristics of the mapping are isolated in $\boldsymbol{\hbar}$ which has a curious geometric interpretation. Since, $\mathbf{x}^{\prime}$ is a homogeneous vector representing a point in an oriented projective plane, $\lambda \mathbf{x}^{\prime}$ represents the same point whenever $\lambda>0$ [9]. Assuming $\lambda=1 / \sqrt{x^{2}+y^{2}+z^{2}}$, it follows from Eq. (1) that

$$
\left\{\begin{array}{l}
x^{\prime}=\frac{x}{\sqrt{x^{2}+y^{2}+z^{2}}} \\
y^{\prime}=\frac{y}{\sqrt{x^{2}+y^{2}+z^{2}}} \\
z^{\prime}-\xi=\frac{z}{\sqrt{x^{2}+y^{2}+z^{2}}}
\end{array}\right.
$$

Assume that $\mathbf{x}$ and $\mathbf{x}^{\prime}$ are projective rays defined in two different coordinates systems in $\mathfrak{R}^{3}$. The origin of the first coordinate system is the effective viewpoint $\mathbf{O}$ and $\mathbf{x}$ is a projective ray going through $\mathbf{O}$. In a similar manner $\mathbf{x}^{\prime}$ represents a projective ray going through the origin $\mathbf{O}^{\prime}$ of the second reference frame. According to the previous equation to each ray $\mathbf{x}$ corresponds one, and only one, projective ray $\mathbf{x}^{\prime}$. The correspondence is such that the pencil of projective rays $\mathbf{x}$ intersects the pencil of rays $\mathbf{x}^{\prime}$ in a set of points lying on a unit sphere centered in $\mathbf{O}$. The equation of the sphere in the coordinate system centered in $\mathbf{O}^{\prime}$ is

$x^{\prime 2}+y^{\prime 2}+\left(z^{\prime}-\xi\right)^{2}=1$

We have just derived the well-known sphere model proposed in [4] (Fig. 2). The homogeneous vector $\mathbf{x}$ can be interpreted as a projective ray joining a 3D point in the scene with the effective projection center $\mathbf{O}$, which intersects the unit sphere in a single point $\mathbf{X}_{\mathbf{m}}$. Consider a point $\mathbf{O}^{\prime}$ in $\mathfrak{R}^{3}$, with coordinates $(X, Y, Z)=(0,0,-\xi)^{\mathrm{T}}$ $(\xi \in[0,1])$. To each $\mathbf{x}$ corresponds an oriented projective ray $\mathbf{x}^{\prime}$, defined by points $\mathbf{O}^{\prime}$ and $\mathbf{X}_{\mathbf{m}}$. The non-linear mapping $\boldsymbol{h}$ is equivalent to projecting the scene on the sphere surface and then re-projecting into a plane from a novel projection center $\mathbf{O}^{\prime}$. Points in the image plane $\mathbf{x}^{\prime \prime}$ are obtained after a collineation $\mathbf{H}_{\mathbf{c}}$ of the $2 \mathrm{D}$ projective points $\mathbf{x}^{\prime}$ (Eq. (2)).

Consider a line in space lying on a plane $\boldsymbol{\Pi}$ with normal $\mathbf{n}=\left(n_{x}, n_{y}, n_{z}\right)^{\mathrm{T}}$ and going through the effective viewpoint $\mathbf{O}$ (Fig. 2). The 3D line is projected in a great circle which is the intersection of $\boldsymbol{\Pi}$ with the reference sphere. Projective rays $\mathbf{x}^{\prime}$, joining $\mathbf{O}^{\prime}$ with points in the great circle, form a central cone with vertex $\mathbf{O}^{\prime}$. The central cone intersects the canonical image plane $\boldsymbol{\Pi}_{\infty}$ in a conic curve $\boldsymbol{\Omega}^{\prime}$. The equation of $\boldsymbol{\Omega}^{\prime}$ is provided in 3 and depends of the normal

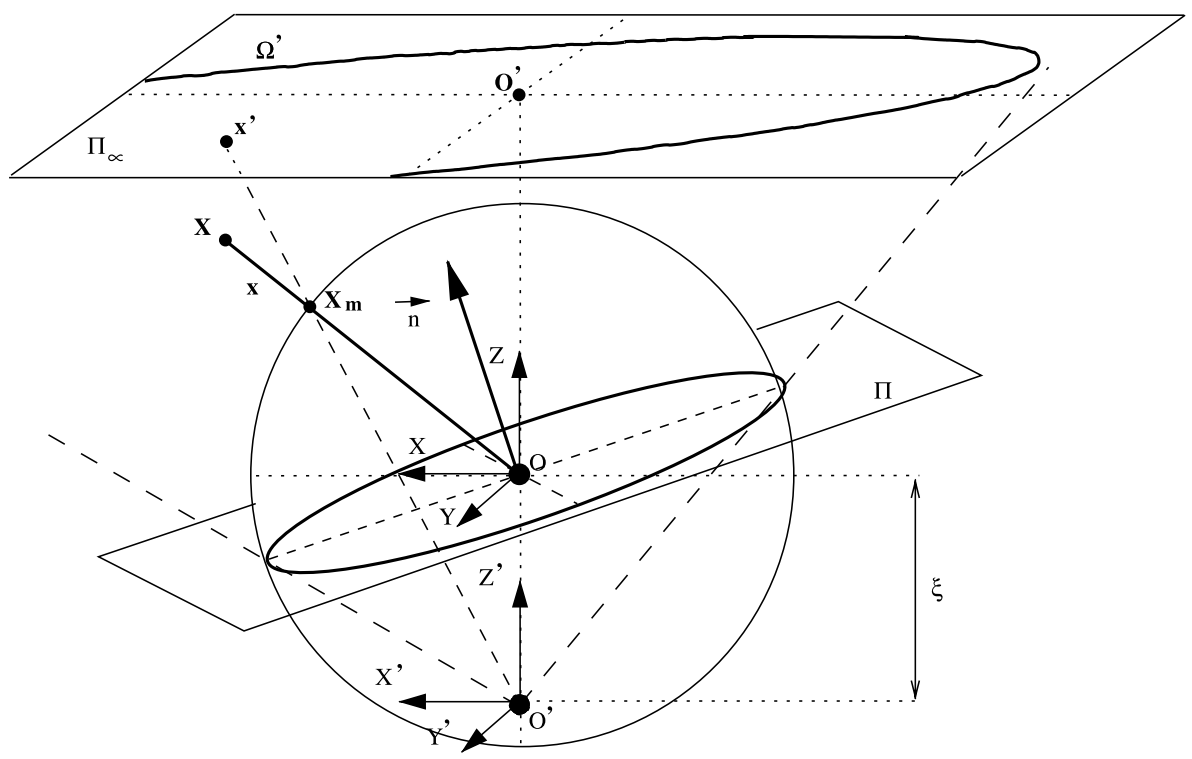

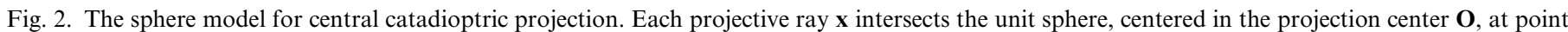
$\mathbf{X}_{\mathrm{m}}$. The new projective point $\mathbf{x}^{\prime}$ is defined by $\mathbf{O}^{\prime}$ and $\mathbf{X}_{\mathrm{m}}$. The distance between the origins $\mathbf{O}$ and $\mathbf{O}^{\prime}$ is $\xi$ which depends on the mirror shape [10]. 
n and parameter $\xi[4,10]$. The original 3D line is projected in the catadioptric image on a conic section $\boldsymbol{\Omega}^{\prime \prime}$, which is the projective transformation of $\boldsymbol{\Omega}^{\prime}\left(\mathbf{\Omega}^{\prime \prime}=\mathbf{H}_{\mathbf{c}}^{-t} \mathbf{\Omega}^{\prime} \mathbf{H}_{\mathrm{c}}^{-1}\right)[4,3]$.

$$
\mathbf{\Omega}^{\prime}=\left[\begin{array}{ccc}
n_{x}^{2}\left(1-\xi^{2}\right)-n_{z}^{2} \xi^{2} & n_{x} n_{y}\left(1-\xi^{2}\right) & n_{x} n_{z} \\
n_{x} n_{y}\left(1-\xi^{2}\right) & n_{y}^{2}\left(1-\xi^{2}\right)-n_{z}^{2} \xi^{2} & n_{y} n_{z} \\
n_{x} n_{z} & n_{y} n_{z} & n_{z}^{2}
\end{array}\right]
$$

Notice that the re-projection center $\mathbf{O}^{\prime}$ depends only on the mirror shape. In the case of parabolic mirrors point $\mathbf{O}^{\prime}$ lies in the sphere surface and the re-projection is a stereographic projection. For hyperbolic systems $\xi \in(0,1)$ and point $\mathbf{O}^{\prime}$ is inside the sphere in the negative $Z$-axis. The conventional perspective camera is a degenerate case of central catadioptric projection where $\xi=0$ and $\mathbf{O}^{\prime}$ is coincident with $\mathbf{O}$.

\subsection{Dioptric systems with radial distortion}

In perspective cameras with lens distortion the mapping between points in the scene and points in the image is no longer described by a linear function. Following the pinhole model, each point in the scene $\mathbf{X}$ originates a projective ray $\mathbf{x}=\mathbf{P X}$ which is transformed into a $2 \mathrm{D}$ projective point $\mathbf{x}^{\prime}=\mathbf{K} \mathbf{x}$. The image point $\mathbf{x}^{\prime \prime}$ is related with $\mathbf{x}^{\prime}$ by a non-linear transformation that models the lens radial distortion. In this paper, we consider the so called division model $[7,8]$ for lens distortion. The non-linear transformation is provided in Eq. (4) with parameter $\xi$ quantifying the amount of radial distortion. Remark that if $\xi=0$ then points $\mathbf{x}^{\prime}$ and $\mathbf{x}^{\prime \prime}$ are the same, and the camera is modeled as a conventional pin-hole. The function of Eq. (4) is the inverse of $\partial$ (see Fig. 1 and Table 1), and isolates the non-linear characteristics of the mapping. In the case of dioptric systems with radial distortion function $\boldsymbol{\hbar}$ is a linear transformation $\mathbf{K}$ (matrix of intrinsic parameters). The division model of Eq. (4) requires that points $\mathbf{x}^{\prime}$ and $\mathbf{x}^{\prime \prime}$ are referenced in a coordinate system with origin in the image distortion center. If the distortion center is not known in advance, we can place it at the image center without significantly affect the correction [6].

$\mathbf{x}^{\prime}=ð^{-1}\left(\mathbf{x}^{\prime \prime}\right)=\left(x^{\prime \prime} z^{\prime \prime}, y^{\prime \prime} z^{\prime \prime}, z^{\prime \prime 2}+\xi\left(x^{\prime \prime 2}+y^{\prime \prime 2}\right)\right)^{\mathrm{T}}$

Transformation $\partial$ has a geometric interpretation similar to the sphere model derived for central catadioptric image formation. As stated before, $\mathbf{x}^{\prime}$ and $\lambda \mathbf{x}^{\prime}$ represent the same point whenever $\lambda$ is a positive scalar [9]. Assuming $\lambda=1 / \sqrt{x^{\prime \prime 2}+y^{\prime \prime 2}}$ in Eq. (4) yields

$\left\{\begin{array}{l}x^{\prime}=\frac{x^{\prime \prime} z^{\prime \prime}}{x^{\prime \prime 2}+y^{\prime \prime 2}} \\ y^{\prime}=\frac{y^{\prime \prime} z^{\prime \prime}}{x^{\prime \prime 2}+y^{\prime \prime 2}} \\ z^{\prime}-\xi=\frac{z^{\prime \prime 2}}{x^{\prime \prime 2}+y^{\prime \prime 2}} .\end{array}\right.$

Reasoning as in the previous section, $\mathbf{x}^{\prime}$ and $\mathbf{x}^{\prime \prime}$ can be interpreted as projective rays going through two distinct origins $\mathbf{O}^{\prime}$ and $\mathbf{O}^{\prime \prime}$. From Eq. (5) follows that the two pencils of rays intersect on a paraboloid with vertex $\mathbf{O}^{\prime \prime}$. The equation of this paraboloid in the coordinate system attached to the origin $\mathbf{O}^{\prime}$ is

$x^{\prime 2}+y^{\prime 2}-\left(z^{\prime}-\xi\right)=0$

The scheme of Fig. 3 is the equivalent of Fig. 2 for the situation of lens with radial distortion. It shows an intuitive 'concrete' model for the non-linear transformation $\partial$ (Table 1) based on the paraboloid derived above. Since in this case the $\xi$ parameter is always negative [8], the effective projection center $\mathbf{O}^{\prime}$ lies inside the parabolic surface. The projective ray $\mathbf{x}^{\prime}$ goes through the viewpoint $\mathbf{O}^{\prime}$ and intersects the

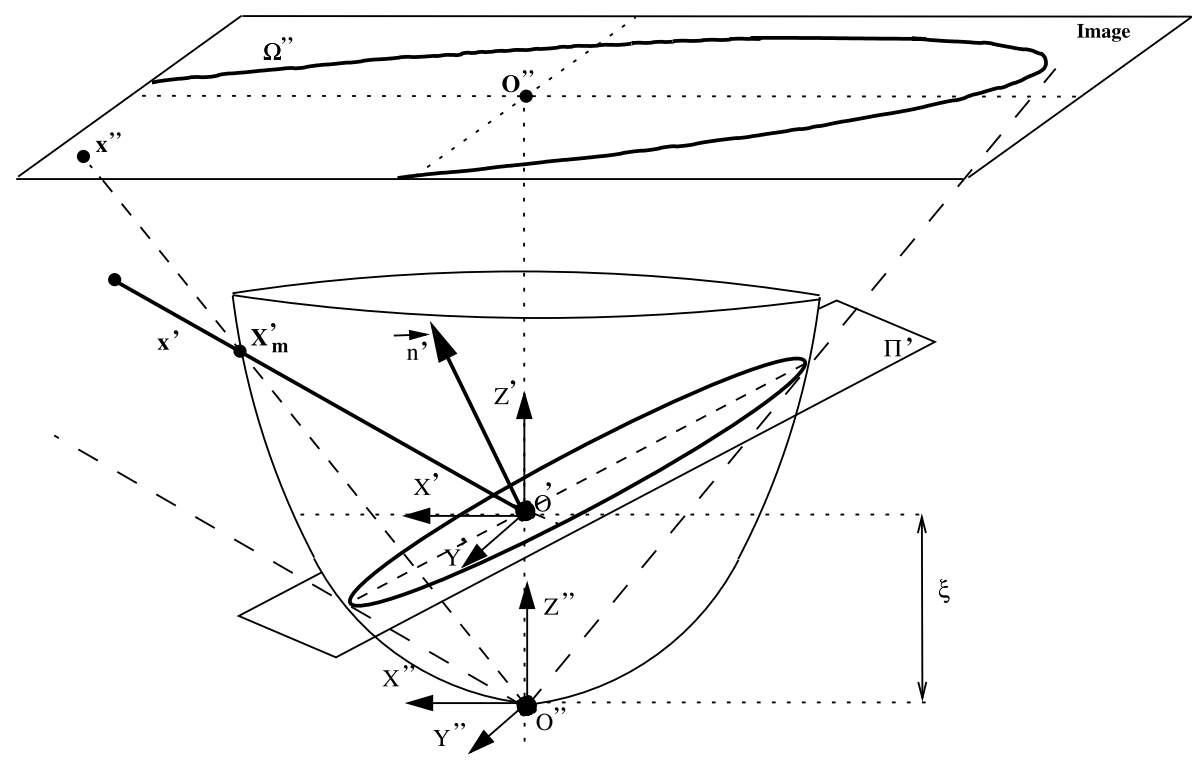

Fig. 3. The paraboloid model for perspective cameras equipped with lens with radial distortion. The division model for lens distortion $[7,8]$ is isomorphic to a projective mapping from a paraboloid to a plane with projection center on the vertex $\mathbf{O}^{\prime \prime}$. The distance between $\mathbf{O}^{\prime \prime}$ and the effective viewpoint is defined by the distortion parameter $\xi$. 
paraboloid at point $\mathbf{X}_{\mathbf{m}}$. By joining $\mathbf{X}_{\mathbf{m}}$ with the vertex $\mathbf{O}^{\prime \prime}$ we obtain the projective ray associated with the distorted image point $\mathbf{x}^{\prime \prime}$. Our model is in accordance to the fact that the effects of radial distortion are more noticeable in the image periphery than in the image center. The reference paraboloid is a quadratic surface in $\wp^{3}$ which is tangent to the plane at infinity at point $\left(X^{\prime}, Y^{\prime}, Z^{\prime}, W^{\prime}\right)^{\mathrm{T}}=$ $(0,0,1,0)^{\mathrm{T}}$. If the angle between the projective ray $\mathbf{x}^{\prime}$ and the $Z^{\prime}$ axis is small, then the intersection point $\mathbf{X}_{\mathbf{m}}$ is close to infinity. In this case the rays associated with $\mathbf{x}^{\prime}$ and $\mathbf{x}^{\prime \prime}$ are almost coincident and the effect of radial distortion is negligible. The paraboloid model herein presented was also derived by Ying et al. [20].

Consider a line in the space that, according to the conventional pin-hole model, is projected into a line $\mathbf{n}^{\prime}=\left(n_{x}^{\prime}, n_{y}^{\prime}, n_{z}^{\prime}\right)^{\mathrm{T}}$ in the projective plane. Points $\mathbf{x}^{\prime}$, lying on line $\mathbf{n}^{\prime}$, are transformed into image points $\mathbf{x}^{\prime \prime}$ by the non-linear function $ð$. Since, $\mathbf{n}^{\prime \mathrm{T}} \mathbf{x}^{\prime}=0$ and $\mathbf{x}^{\prime}=\chi^{-1}\left(\mathbf{x}^{\prime \prime}\right)$, then $\mathbf{n}^{T} \partial^{-1}\left(\mathbf{x}^{\prime \prime}\right)=0$. After some algebraic manipulation the previous equality can be written in the form $\mathbf{x}^{\prime \prime} \boldsymbol{\Omega}^{\prime \prime} \mathbf{x}^{\prime \prime}=0$ with $\boldsymbol{\Omega}^{\prime \prime}$ given by Eq. (6). In a similar way to what happens for the central catadioptric systems, the non-linear mapping $\partial$ transforms lines $\mathbf{n}^{\prime}$ into a conic sections $\boldsymbol{\Omega}^{\prime \prime}$ (see Fig. 3).

$$
\boldsymbol{\Omega}^{\prime \prime}=\left[\begin{array}{ccc}
\xi n_{z}^{\prime} & 0 & \frac{n_{x}^{\prime}}{2} \\
0 & \xi n_{z}^{\prime} & \frac{n_{y}^{\prime}}{2} \\
\frac{n_{x}^{\prime}}{2} & \frac{n_{y}^{\prime}}{2} & n_{z}^{\prime}
\end{array}\right]
$$

\section{Embedding $\wp^{2}$ into $\wp^{5}$ using Veronese maps}

Perspective projection can be formulated as a transformation of $\mathfrak{R}^{3}$ into $\mathfrak{R}^{2}$. Points $\mathbf{X}=(X, Y, Z)^{\mathrm{T}}$ are mapped into points $\mathbf{x}=(x, y)^{\mathrm{T}}$ by a non-linear function $f(\mathbf{X})=$ $(X / Z, Y / Z)^{\mathrm{T}}$. A standard technique used in algebra to render a nonlinear problem into a linear one is to find an embedding that lifts the problem into a higher dimensional space. For conventional cameras, the additional homogeneous coordinate linearizes the mapping function and simplifies most of the mathematic relations. In the previous section we established a unifying model that includes central catadioptric sensors and lens with radial distortion. Unfortunately the use of an additional homogeneous coordinate does no longer suffice to cope with the non-linearities in the image formation.

In this paper, we propose the embedding of the projective plane into a higher dimensional space in order to study the geometry of general single viewpoint images in a unified framework. This idea has already been explored by other authors to solve several computer vision problems. Higher-dimensional projection matrices are proposed in [11] for the representation of various applications where the world is no longer rigid. In [12], lifted coordinates are used to obtain a fundamental matrix between paracatadioptric views. Sturm generalizes this framework to analyze the relations between multiple views of a static scene where the views are taken by any mixture of paracatadioptric, perspective or affine cameras [13].

The present section discusses the embedding of the projective plane $\wp^{2}$ in $\wp^{5}$ (Eq. (7)) using Veronese mapping $[14,15]$. This polynomial embedding preserves homogeneity and is suitable to represent quadratic relations between image points [16,17]. Moreover there is a natural duality between lifted points $\widetilde{\mathbf{x}}$ and conics which is advantageous when dealing with catadioptric and distorted projection of lines. It is also shown that projective transformations in $\wp^{2}$ can be transposed to $\wp^{5}$ in a straightforward manner.

$\mathbf{x} \in \wp^{2} \rightarrow \widetilde{\mathbf{x}}=\left(x_{0}, x_{1}, x_{2}, x_{3}, x_{4}, x_{5}\right)^{\mathrm{T}} \in \wp^{5}$

\subsection{Lifting point coordinates}

Consider an operator $\boldsymbol{\Gamma}$ which transforms two $3 \times 1$ vectors $\mathbf{x}, \overline{\mathbf{x}}$ into a $6 \times 1$ vector as shown in Eq. (8)

$\boldsymbol{\Gamma}(\mathbf{x}, \overline{\mathbf{x}})=\left(x \bar{x}, \frac{x \bar{y}+y \bar{x}}{2}, y \bar{y}, \frac{x \bar{x}+z \bar{x}}{2}, \frac{y \bar{z}+z \bar{y}}{2}, z \bar{z}\right)^{\mathrm{T}}$

The operator $\Gamma$ can be used to map pairs of points in the projective plane $\wp^{2}$, with homogeneous coordinates $\mathbf{x}$ and $\overline{\mathbf{x}}$, into points in the $5 \mathrm{D}$ projective space $\wp^{5}$. To each pair of points $\mathbf{x}, \overline{\mathbf{x}}$ corresponds one, and only one, point $\widetilde{\mathbf{x}}=\boldsymbol{\Gamma}(\mathbf{x}, \overline{\mathbf{x}})$ which lies on a primal $\mathbf{S}$ called the cubic symmetroid [14]. The cubic symmetroid $\mathbf{S}$ is a non-linear subset of $\wp^{5}$ defined by the following equation:

$x_{0} x_{2} x_{5}+2 x_{1} x_{3} x_{4}-x_{0} x_{4}^{2}-x_{2} x_{3}^{2}-x_{5} x_{1}^{2}=0, \quad \forall_{\tilde{\mathbf{x}} \in \mathbf{S}}$

By making $\overline{\mathbf{x}}=\mathbf{x}$ the operator $\Gamma$ can be used to map a single point in $\wp^{2}$ into a point in $\wp^{5}$. In this case the lifting function becomes

$\mathbf{x} \rightarrow \widetilde{\mathbf{x}}=\boldsymbol{\Gamma}(\mathbf{x}, \mathbf{x})=\left(x^{2}, x y, y^{2}, x z, y z, z^{2}\right)^{\mathrm{T}}$.

To each point $\mathbf{x}$ in the projective plane corresponds one, and only one, point $\widetilde{\mathbf{x}}$ lying on a quadratic surface $\mathbf{V}$ in $\wp^{5}$. This surface, defined by the triplet of Eq. (11), is called the Veronese surface and is a sub-set of the cubic symmetroid $\mathbf{S}[14,15]$. The mapping of Eq. (10) is the second order Veronese mapping that will be used to embed the projective plane $\wp^{2}$ into the $5 \mathrm{D}$ projective space.

$x_{1}^{2}-x_{0} x_{2}=0 \wedge x_{3}^{2}-x_{0} x_{5}=0 \wedge x_{4}^{2}-x_{2} x_{5}=0, \quad \forall_{\widetilde{\mathbf{x}} \in \mathbf{V}}$.

\subsection{Lifting lines and conics}

A conic curve in the projective plane $\wp^{2}$ is usually represented by a $3 \times 3$ symmetric matrix $\boldsymbol{\Omega}$. Point $\mathbf{x}$ lies on the conic if, and only if, equation $\mathbf{x}^{\mathrm{T}} \boldsymbol{\Omega} \mathbf{x}=0$ is satisfied. Since a $3 \times 3$ symmetric matrix has 6 parameters, the conic locus can also be represented by a $6 \times 1$ homogeneous vector $\widetilde{\boldsymbol{\omega}}$ (Eq. (12)). Vector $\widetilde{\boldsymbol{\omega}}$ is the representation in lifted coordinates of the planar conic $\mathbf{\Omega}$ 


$$
\mathbf{\Omega}=\left[\begin{array}{lll}
a & b & d \\
b & c & e \\
d & e & f
\end{array}\right] \rightarrow \widetilde{\boldsymbol{\omega}}=(a, 2 b, c, 2 d, 2 e, f)^{\mathrm{T}} .
$$

Point $\mathbf{x}$ lies on the conic locus $\boldsymbol{\Omega}$ if, and only if, its lifted coordinates $\widetilde{\mathbf{x}}$ are orthogonal to vector $\widetilde{\boldsymbol{\omega}}\left(\widetilde{\boldsymbol{\omega}}^{\mathrm{T}} \cdot \widetilde{\mathbf{x}}=0\right)$. Moreover, if points $\mathbf{x}$ and $\overline{\mathbf{x}}$ are harmonic conjugates with respect to the conic then $\mathbf{x}^{\mathrm{T}} \boldsymbol{\Omega} \overline{\mathbf{x}}=0$ and $\widetilde{\boldsymbol{\omega}}^{\mathrm{T}} . \boldsymbol{\Gamma}(\mathbf{x}, \overline{\mathbf{x}})=0$. In the same way as points and lines are dual entities in $\wp^{2}$, there is a duality between points and conics in the lifted space $\wp^{5}$. Since the general single viewpoint image of a line is a conic (Eqs. 3 and 6), this duality will prove to be a nice and useful property.

Conic $\boldsymbol{\Omega}=\mathbf{m} \cdot \mathbf{l}^{\mathrm{T}}+\mathbf{l} \cdot \mathbf{m}^{\mathrm{T}}$ is composed of two lines $\mathbf{m}$ and $\mathbf{l}$ lying on the projective plane $\wp^{2}$. In this case, the conic is said to be degenerate, the $3 \times 3$ symmetric matrix $\Omega$ is rank 2, and Eq. (12) becomes

$$
\mathbf{\Omega}=\mathbf{m l}^{\mathrm{T}}+\mathbf{l m}^{\mathrm{T}} \rightarrow \widetilde{\boldsymbol{\omega}}=\underbrace{\left[\begin{array}{llllll}
1 & 0 & 0 & 0 & 0 & 0 \\
0 & 2 & 0 & 0 & 0 & 0 \\
0 & 0 & 1 & 0 & 0 & 0 \\
0 & 0 & 0 & 2 & 0 & 0 \\
0 & 0 & 0 & 0 & 2 & 0 \\
0 & 0 & 0 & 0 & 0 & 1
\end{array}\right]}_{\widetilde{\mathbf{D}}} . \boldsymbol{\Gamma}(\mathbf{m}, \mathbf{l})
$$

In a similar way a conic locus can be composed by a single line $\mathbf{n}=\left(n_{x}, n_{y}, n_{z}\right)^{\mathrm{T}}$. Matrix $\boldsymbol{\Omega}=\mathbf{n} \cdot \mathbf{n}^{\mathrm{T}}$ has rank 1 and the result of Eq. (12) can be used to establish the lifted representation of a line

$\mathbf{n} \rightarrow \widetilde{\mathbf{n}}=\widetilde{\mathbf{D}} \cdot \boldsymbol{\Gamma}(\mathbf{n}, \mathbf{n})=\left(n_{x}^{2}, 2 n_{x} n_{y}, n_{y}^{2}, 2 n_{x} n_{z}, 2 n_{y} n_{z}, n_{z}^{2}\right)^{\mathrm{T}}$

Consider a point $\mathbf{x}$ in $\wp^{2}$ lying on line $\mathbf{n}$ such that $\mathbf{n}^{\mathrm{T}} \cdot \mathbf{x}=0$. Point $\mathbf{x}$ is on the line if, and only if, its lifted coordinates $\tilde{\mathbf{n}}$ are orthogonal to the homogeneous vector $\widetilde{\mathbf{n}}\left(\widetilde{\mathbf{n}}^{\mathrm{T}} \widetilde{\mathbf{x}}=0\right)$. Points and lines are dual entities in $\wp^{2}$ as well as in the lifted space $\wp^{5}$. By embedding the projective plane into $\wp^{5}$ lines and conics are treated in a uniform manner. The duality between points and lines is preserved and extended for the case of points and conics. The space of all conics is the dual 5D projective space $\wp^{5 *}$, because each point $\widetilde{\omega}$ corresponds to a conic curve $\boldsymbol{\Omega}$ in the original $2 \mathrm{D}$ plane. The set of all lines $\mathbf{n}$ is mapped into a non-linear subset $\mathbf{V}^{*}$ of $\wp^{5 *}$, which is the projective transformation of the Veronese surface $\mathbf{V}$ by $\widetilde{\mathbf{D}}$ (Eq. (14)).

\subsection{Lifting linear transformations}

In the previous sections, we discussed the representation of points, lines and conics in the $5 \mathrm{D}$ projective space $\wp^{5}$. However a geometry is defined not only by a set of objects but also by the group of transformations acting on them [18]. This section shows how a linear transformation on the original space $\wp^{2}$ can be coherently transferred to the lifted space $\wp^{5}$.
Consider a linear transformation, represented by a $3 \times 3$ matrix $\mathbf{H}$, which maps any two points $\mathbf{x}$ and $\overline{\mathbf{x}}$ into points $\mathbf{H x}$ and $\mathbf{H} \overline{\mathbf{x}}$. Both pairs of points can be lifted to $\wp^{5}$ using the operator $\boldsymbol{\Gamma}$ of Eq. (8). We wish to obtain a new operator $\boldsymbol{\Lambda}$ that has the following characteristic

$\boldsymbol{\Gamma}(\mathbf{H x}, \mathbf{H} \overline{\mathbf{x}})=\boldsymbol{\Lambda}(\mathbf{H}) . \boldsymbol{\Gamma}(\mathbf{x}, \overline{\mathbf{x}})$

The desired result can be derived by developing Eq. (15) and performing some algebraic manipulation. The operator $\Lambda$, transforming a $3 \times 3$ matrix $\mathbf{H}$ into a $6 \times 6$ matrix $\widetilde{\mathbf{H}}$, is provided in Eq. (16) with $\mathbf{v}_{1}, \mathbf{v}_{2}$ and $\mathbf{v}_{3}$ denoting the columns of the original matrix $\mathbf{H}$.

$$
\boldsymbol{\Lambda}(\underbrace{\left[\mathbf{v}_{1} \mathbf{v}_{2} \mathbf{v}_{3}\right]}_{\mathbf{H}})=\underbrace{\left[\begin{array}{c}
\boldsymbol{\Gamma}\left(\mathbf{v}_{1}, \mathbf{v}_{1}\right)^{\mathrm{T}} \\
\boldsymbol{\Gamma}\left(\mathbf{v}_{1}, \mathbf{v}_{2}\right)^{\mathrm{T}} \\
\boldsymbol{\Gamma}\left(\mathbf{v}_{2}, \mathbf{v}_{2}\right)^{\mathrm{T}} \\
\boldsymbol{\Gamma}\left(\mathbf{v}_{1}, \mathbf{v}_{3}\right)^{\mathrm{T}} \\
\boldsymbol{\Gamma}\left(\mathbf{v}_{2}, \mathbf{v}_{3}\right)^{\mathrm{T}} \\
\boldsymbol{\Gamma}\left(\mathbf{v}_{3}, \mathbf{v}_{3}\right)^{\mathrm{T}}
\end{array}\right]}_{\widetilde{\mathbf{H}}} \widetilde{\mathbf{D}}
$$

It can be proved that $\boldsymbol{\Lambda}$, not only satisfies the relation stated on Eq. (15), but also has the following properties

$$
\begin{aligned}
\boldsymbol{\Lambda}\left(\mathbf{H}^{-1}\right) & =\boldsymbol{\Lambda}(\mathbf{H})^{-1} \\
\boldsymbol{\Lambda}(\mathbf{H} . \mathbf{B}) & =\boldsymbol{\Lambda}(\mathbf{H}) \cdot \boldsymbol{\Lambda}(\mathbf{B}) \\
\boldsymbol{\Lambda}\left(\mathbf{H}^{\mathrm{T}}\right) & =\widetilde{\mathbf{D}}^{-1} \cdot \boldsymbol{\Lambda}(\mathbf{H})^{\mathrm{T}} \cdot \widetilde{\mathbf{D}} \\
\boldsymbol{\Lambda}\left(\mathbf{I}_{3 \times 3}\right) & =\mathbf{I}_{6 \times 6}
\end{aligned}
$$

From Eq. (15) comes that if $\mathbf{x}$ and $\mathbf{y}$ are two points in $\wp^{2}$ such that $\mathbf{y}=\mathbf{H x}$ then $\widetilde{\mathbf{y}}=\boldsymbol{\Lambda}(\mathbf{H}) \cdot \widetilde{\mathbf{x}}$ where $\widetilde{\mathbf{x}}$ and $\widetilde{\mathbf{y}}$ are the lifted coordinates of the points. The operator $\boldsymbol{\Lambda}$ maps the linear transformation $\mathbf{H}$ in the plane into the linear transformation $\widetilde{\mathbf{H}}=\boldsymbol{\Lambda}(\mathbf{H})$ in $\wp^{5}$. The transformation of points and conics are transferred to the $5 \mathrm{D}$ projective space in the following manner

$\mathbf{y}=\mathbf{H x} \rightarrow \widetilde{\mathbf{y}}=\widetilde{\mathbf{H}} \widetilde{\mathbf{x}}$

$\boldsymbol{\Psi}=\mathbf{H}^{-t} \mathbf{\Omega} \mathbf{H}^{-1} \rightarrow \widetilde{\boldsymbol{\psi}}=\widetilde{\mathbf{H}}^{-t} \widetilde{\boldsymbol{\omega}}$

We just proved that the set of linear transformations in $\wp^{2}$ can be mapped into a subset of linear transformations in $\wp^{5}$. Any transformation, represented by a singular or non-singular $3 \times 3$ matrix $\mathbf{H}$, has a correspondence in $\widetilde{\mathbf{H}}=\boldsymbol{\Lambda}(\mathbf{H})$. However note that there are linear transformations in $\wp^{5}$ without any correspondence in the projective plane.

\section{The subset of line images}

This section applies the established framework in order to study the properties of line projection in central catadioptric systems and cameras with radial distortion. If it is true that a line is mapped into a conic in the image, it is not true that any conic can be the projection of a line. It is shown that a conic section $\widetilde{\omega}$ is the projection of a line 
if, and only if, it lies in a certain subset of $\wp^{5}$ defined by the sensor type and calibration. This subset is a linear subspace for paracatadioptric cameras and cameras with radial distortion, and a quadratic surface for hyperbolic systems.

\subsection{Central catadioptric projection of lines}

Assume that a certain line in the world is projected into a conic section $\boldsymbol{\Omega}^{\prime \prime}$ in the catadioptric image plane. As shown in Fig. 2, the line lies in plane $\Pi$ that contains the projection center $\mathbf{O}$ and is orthogonal to $\mathbf{n}=\left(n_{x}, n_{y}, n_{z}\right)^{\mathrm{T}}$. The catadioptric projection of the line is $\boldsymbol{\Omega}^{\prime \prime}=\mathbf{H}_{\mathbf{c}}^{-t} \mathbf{\Omega}^{\prime} \mathbf{H}_{\mathbf{c}}^{-1}$ with $\mathbf{H}_{\mathbf{c}}$ the calibration matrix. The conic $\boldsymbol{\Omega}^{\prime}$ is provided in Eq. (3) and depends on the normal $\mathbf{n}$ and the shape of the mirror.

The framework derived in the previous section is now used to transpose to $\wp^{5}$ the model for line projection discussed in Section 2.1. Conic $\boldsymbol{\Omega}^{\prime}$ is mapped into $\boldsymbol{\omega}^{\prime}$ in the 5D projective space. As shown in Eq. (3) the conic depends on the normal $\mathbf{n}$ and on parameter $\xi$. This dependence can be represented in $\wp^{5}$ by $\widetilde{\boldsymbol{\omega}}^{\prime}=\widetilde{\boldsymbol{\Delta}}_{\mathrm{c}} \widetilde{\mathbf{n}}$ with $\widetilde{\boldsymbol{\Delta}}_{\mathrm{c}}$ given by Eq. (19). The lifted coordinates of the final image of the line are $\widetilde{\boldsymbol{\omega}} / \prime=\widetilde{\mathbf{H}}_{\mathbf{c}} \widetilde{\boldsymbol{\Delta}}_{\mathbf{c}} \widetilde{\mathbf{n}}$. Hence forth, if nothing is said, the collineation $\widetilde{\mathbf{H}}_{\mathbf{c}}$ is ignored and we will work directly with $\widetilde{\boldsymbol{\omega}}^{\prime}=\widetilde{\mathbf{H}}_{\mathbf{c}}^{-1} \widetilde{\boldsymbol{\omega}}^{\prime \prime}$.

$\underbrace{\left[\begin{array}{c}a^{\prime} \\ 2 b^{\prime} \\ c^{\prime} \\ 2 d^{\prime} \\ 2 e^{\prime} \\ f^{\prime}\end{array}\right]}_{\widetilde{\omega^{\prime}}}=\underbrace{\left[\begin{array}{cccccc}1-\xi^{2} & 0 & 0 & 0 & 0 & -\xi^{2} \\ 0 & 1-\xi^{2} & 0 & 0 & 0 & 0 \\ 0 & 0 & 1-\xi^{2} & 0 & 0 & -\xi^{2} \\ 0 & 0 & 0 & 1 & 0 & 0 \\ 0 & 0 & 0 & 0 & 1 & 0 \\ 0 & 0 & 0 & 0 & 0 & 1\end{array}\right]}_{\widetilde{\Delta}_{\mathrm{c}}} \underbrace{\left[\begin{array}{c}n_{x}^{2} \\ 2 n_{x} n_{y} \\ n_{y}^{2} \\ 2 n_{x} n_{z} \\ 2 n_{y} n_{z} \\ n_{z}^{2}\end{array}\right]}_{\widetilde{\mathbf{n}}}$

Notice that the linear transformation $\widetilde{\boldsymbol{\Delta}}_{\mathrm{c}}$, derived from Eq. (3), does not have an equivalent transformation in the projective plane (Eq. (16)). The catadioptric projection of a line, despite of being non-linear in $\wp^{2}$, is described by a linear relation in $\wp^{5}$.

As stated in Section 3.2, a line $\mathbf{n}$ in the projective plane is lifted into a point $\widetilde{\mathbf{n}}$ which lies on the quadratic surface $\mathbf{V}^{*}$ in $\wp^{5 *}$. From Eq. (19) it follows that a generic conic $\widetilde{\boldsymbol{\omega}}^{\prime}$ is the catadioptric projection of a line if, and only if, $\widetilde{\boldsymbol{\Delta}}_{\mathrm{c}}^{-1} \widetilde{\boldsymbol{\omega}}^{\prime} \in \mathbf{V}^{*}$. Since surface $\mathbf{V}^{*}$ is the projective transformation of the Veronese surface $\mathbf{V}$ (Eq. (11)) by $\widetilde{\mathbf{D}}$, then $\widetilde{\boldsymbol{\omega}}^{\prime}=\left(a^{\prime}, 2 b^{\prime}, c^{\prime}, 2 d^{\prime}, 2 e^{\prime}, f^{\prime}\right)^{\mathrm{T}}$ is the projection of a line if, and only if,

$$
\left\{\begin{array}{l}
d^{\prime 2}\left(1-\xi^{2}\right)-f^{\prime}\left(a^{\prime}+f^{\prime} \xi^{2}\right)=0 \\
e^{\prime 2}\left(1-\xi^{2}\right)-f^{\prime}\left(c^{\prime}+f^{\prime} \xi^{2}\right)=0, \quad \forall \widetilde{\omega^{\prime} \in \zeta} \\
b^{\prime 2}-\left(a^{\prime}+f \xi^{2}\right)\left(c^{\prime}+f^{\prime} \xi^{2}\right)=0
\end{array}\right.
$$

Eq. (20) defines a quadratic surface $\zeta$ in the space of all conics. The constraints of Eq. (20) have been recently introduced in [19] and used as invariants for calibration purposes.

\subsection{Line projection in paracatadioptric cameras}

Let's consider the situation of paracatadioptric cameras where $\xi=1$. In this case point $\mathbf{O}^{\prime}$ lies on the sphere surface (Fig. 2) and the re-projection from the sphere to the plane becomes a stereographic projection [4]. Eq. (21) is derived from Eq. (20) by making $\xi=1$. For the particular case of paracatadioptric cameras the quadratic surface $\zeta$ degenerates into a linear subspace $\varphi$ which is the set of all line projections $\widetilde{\boldsymbol{\omega}}^{\prime}$.

$a^{\prime}+f^{\prime}=0 \wedge c^{\prime}+f^{\prime}=0 \wedge b^{\prime 2}=0, \quad \forall \underset{\omega^{\prime} \in \varphi}{\sim}$

Stating this result in a different manner, the conic $\mathbf{\Omega}^{\prime}$ is the paracatadioptric projection of a line if, and only if, the corresponding lifted representation $\widetilde{\boldsymbol{\omega}}^{\prime}$ is on the null space of matrix $\mathbf{N}_{\mathbf{p}}$.

$$
\underbrace{\left[\begin{array}{llllll}
1 & 0 & 0 & 0 & 0 & 1 \\
0 & 0 & 1 & 0 & 0 & 1 \\
0 & 1 & 0 & 0 & 0 & 0
\end{array}\right]}_{\mathbf{N}_{\mathbf{p}}} \widetilde{\boldsymbol{\omega}}^{\prime}=0
$$

We have already seen that if point $\mathbf{x}^{\prime}$ is on conic $\boldsymbol{\Omega}^{\prime}$ then $\widetilde{\boldsymbol{\omega}}^{\prime T} \widetilde{\mathbf{x}}^{\prime}=0$. The lifted point $\widetilde{\mathbf{x}}^{\prime}$ must lie on the prime of $\wp^{5}$ that is orthogonal to $\widetilde{\boldsymbol{\omega}}^{\prime}[14]$. However, not all points in this prime are lifted coordinates of points in $\wp^{2}$. Section 3.1 shows that only points lying on the Veronese surface $\mathbf{V}$ have a correspondence on the projective plane. Thus, points $\mathbf{x}^{\prime}$ lying on $\mathbf{\Omega}^{\prime}$ are mapped into a subset of $\wp^{5}$ defined by the intersection of the prime orthogonal to $\widetilde{\boldsymbol{\omega}}^{\prime}$ with the Veronese surface $\mathbf{V}$.

Consider the set of all conic sections $\boldsymbol{\Omega}^{\prime}$ corresponding to paracatadioptic line projections. If this conic set has a common point $\mathbf{x}^{\prime}$ then its lifted vector $\widetilde{\mathbf{x}}^{\prime}$ must be on the intersection of $\mathbf{V}$ with the hyperplane orthogonal to $\boldsymbol{\varphi}$. Points $\widetilde{\mathbf{I}}^{\prime}$ and $\widetilde{\mathbf{J}}^{\prime}$ are computed by intersecting the range of matrix $\mathbf{N}_{\mathbf{p}}^{\mathrm{T}}$ (the orthogonal hyperplane) with the Veronese surface defined in Eq. (11). These points are the lifted coordinates of the circular points in the projective plane where all paracatadioptric line images $\boldsymbol{\Omega}^{\prime}$ intersect.

$$
\left\{\begin{array} { l } 
{ \widetilde { \mathbf { I } } ^ { \prime } = ( 1 , \mathrm { i } , - 1 , 0 , 0 , 0 ) ^ { \mathrm { T } } } \\
{ \widetilde { \mathbf { J } } ^ { \prime } = ( 1 , - \mathrm { i } , - 1 , 0 , 0 , 0 ) ^ { \mathrm { T } } }
\end{array} \rightarrow \left\{\begin{array}{l}
\mathbf{I}^{\prime}=(1, \mathrm{i}, 0)^{\mathrm{T}} \\
\mathbf{J}^{\prime}=(1,-\mathrm{i}, 0)^{\mathrm{T}}
\end{array}\right.\right.
$$

In a similar way, if there is a pair of points $\mathbf{x}, \overline{\mathbf{x}}$ that are harmonic conjugate with respect to all conics $\boldsymbol{\Omega}^{\prime}$ then, the corresponding vector $\boldsymbol{\Gamma}(\mathbf{x}, \overline{\mathbf{x}})$, must be in the intersection of $\mathbf{S}$ with the range of $\mathbf{N}_{\mathbf{p}}^{\mathrm{T}}$. The intersection can be determined from Eqs. 9 and 22 defining the cubic symmetroid $\mathbf{S}$ and matrix $\mathbf{N}_{\mathbf{p}}$. The result is presented in Eq. (24) where $\lambda$ is a free scalar.

$$
\left\{\begin{array}{l}
\widetilde{\mathbf{P}^{\prime} Q^{\prime}}=(-\lambda, 1, \lambda, 0,0,0)^{\mathrm{T}} \rightarrow\left\{\begin{array}{l}
\mathbf{P}^{\prime}=\left(1+\sqrt{1+\lambda^{2}}, \lambda, 0\right)^{\mathrm{T}} \\
\mathbf{Q}^{\prime}=\left(1-\sqrt{1+\lambda^{2}}, \lambda, 0\right)^{\mathrm{T}}
\end{array}\right. \\
\widetilde{\mathbf{R}^{\prime} T^{\prime}}=\left(1, \lambda, \lambda^{2}, 0,0,1+\lambda^{2}\right)^{\mathrm{T}} \rightarrow\left\{\begin{array}{l}
\mathbf{R}^{\prime}=\left(1, \lambda,-\mathrm{i} \sqrt{1+\lambda^{2}}\right)^{\mathrm{T}} \\
\mathbf{T}^{\prime}=\left(1, \lambda, \mathrm{i} \sqrt{1+\lambda^{2}}\right)^{\mathrm{T}}
\end{array}\right.
\end{array}\right.
$$


According to Eq. (23), any paracatadioptric projection of a line must go through the circular points. This is not surprising, since the stereographic projection of a great circle is always a circle (see Fig. 2). However, not all circles correspond to the projection of lines. While points $\mathbf{P}^{\prime}, \mathbf{Q}^{\prime}$ are harmonic conjugate with respect to a all circles, the same does not happen with the pair $\mathbf{R}^{\prime}, \mathbf{T}^{\prime}$. Thus, a conic $\boldsymbol{\Omega}^{\prime}$ is the paracatadioptric image of a line if, and only if, it goes through the circular points and satisfies $\mathbf{R}^{\prime T} \mathbf{\Omega}^{\prime} \mathbf{T}^{\prime}=0$. This result has been used in $[21,22]$ in order to constrain the search space and accurately estimate line projections in the paracatadioptric image plane.

\subsection{Line projection in cameras with radial distortion}

We have already shown that for catadioptric cameras the model for line projection becomes linear when the projective plane is embedded in $\wp^{5}$. A similar derivation can be applied to dioptric cameras with radial distortion. According to the conventional pin-hole model a line in the scene is mapped into a line $\mathbf{n}^{\prime}$ in the image plane. However, and as discussed on Section 2.2, the non-linear effect of radial distortion transforms $\mathbf{n}^{\prime}$ into a conic curve $\boldsymbol{\Omega}^{\prime \prime}$. If $\widetilde{\boldsymbol{\omega}}^{\prime \prime}$ and $\widetilde{\mathbf{n}}^{\prime}$ are the 5D representations of $\boldsymbol{\Omega}^{\prime \prime}$ and $\mathbf{n}^{\prime}$ it follows from Eq. (6) that

$$
\underbrace{\left[\begin{array}{c}
a^{\prime \prime} \\
2 b^{\prime \prime} \\
c^{\prime \prime} \\
2 d^{\prime \prime} \\
2 e^{\prime \prime} \\
f^{\prime \prime}
\end{array}\right]}_{\widetilde{\omega}^{\prime \prime}}=\underbrace{\left[\begin{array}{cccccc}
0 & 0 & 0 & 0 & 0 & \xi \\
0 & 0 & 0 & 0 & 0 & 0 \\
0 & 0 & 0 & 0 & 0 & \xi \\
0 & 0 & 0 & 0.5 & 0 & 0 \\
0 & 0 & 0 & 0 & 0.5 & 0 \\
0 & 0 & 0 & 0 & 0 & 1
\end{array}\right]}_{\widetilde{\boldsymbol{\Delta}}_{r}} \underbrace{\left[\begin{array}{c}
n_{x}^{\prime 2} \\
2 n_{x}^{\prime} n_{y}^{\prime} \\
n_{y}^{\prime 2} \\
2 n_{x}^{\prime} n_{z}^{\prime} \\
2 n_{y}^{\prime} n_{z}^{\prime} \\
n_{z}^{\prime 2}
\end{array}\right]}_{\widetilde{\mathbf{n}^{\prime}}}
$$

For the paracatadioptric camera situation $(\xi=1)$ matrix $\widetilde{\boldsymbol{\Delta}}_{\mathrm{c}}$ has a structure similar to matrix $\widetilde{\boldsymbol{\Delta}}_{r}$. It can be proved that a conic section $\widetilde{\omega} / \prime$ is the distorted projection of a line if, and only if, it lies on a hyperplane $\varsigma$ defined as follows $a^{\prime \prime}-\xi f^{\prime \prime}=0 \wedge c^{\prime \prime}-\xi f^{\prime \prime}=0 \wedge b^{\prime \prime 2}=0, \forall{\widetilde{\omega^{\prime} \in \varsigma}}$

Repeating the reasoning that we did for the paracatadioptric camera, it can be shown that conic $\boldsymbol{\Omega}^{\prime \prime}$ is the distorted projection of a line if, and only if, it goes through the circular points of Eq. (23) and satisfies the condition $\mathbf{M}^{\prime \prime} \boldsymbol{\Omega}^{\prime \prime} \mathbf{N}^{\prime \prime}=0$ with $\mathbf{M}^{\prime \prime}$ and $\mathbf{N}^{\prime \prime}$ given below

$$
\widetilde{\mathbf{M}^{\prime \prime} N^{\prime \prime}}=\left(1, \lambda, \lambda^{2}, 0,0,-\xi\left(1+\lambda^{2}\right)\right)^{\mathrm{T}} \rightarrow\left\{\begin{array}{l}
\mathbf{M}^{\prime \prime}=\left(1, \lambda, \sqrt{\xi\left(1+\lambda^{2}\right)}\right)^{\mathrm{T}} \\
\mathbf{N}^{\prime \prime}=\left(1, \lambda,-\sqrt{\xi\left(1+\lambda^{2}\right)}\right)^{\mathrm{T}}
\end{array}\right.
$$

\section{Experiments}

Conventional perspective cameras, central catadioptric systems and dioptric cameras with lens distortion underly a common model for central projection (Fig. 1). According to this model the central projection of a line is in general a conic curve that is dual to the lifted representation of image points (Section 3). The embedding of the projective plane into $\wp^{5}$ provides useful geometric insights and shows the similarities between the different types of sensors.

We proved that in the case of paracatadioptric systems the lifted representation of a line projection lies in a plane in the dual space $\wp^{5 *}$. This fact is explored in [21] in order to calibrate the sensor using the image of lines. A similar approach can be applied for the estimation of lens distortion. Consider the projection of a line $\mathbf{n}^{\prime}=\left(n_{x}^{\prime}, n_{y}^{\prime}, n_{z}^{\prime}\right)^{\mathrm{T}}$ in a camera with radial distortion. From Eq. (6) follows that, if $\mathbf{n}^{\prime}$ does not go through the distortion center $\left(n_{z}^{\prime} \neq 0\right)$, then its projection is a conic curve with lifted representation

$$
\widetilde{\boldsymbol{\omega}}^{\prime \prime}=\left(\xi, 0, \xi, \frac{n_{x}^{\prime}}{n_{z}^{\prime}}, \frac{n_{y}^{\prime}}{n_{z}^{\prime}}, 1\right)^{\mathrm{T}}
$$

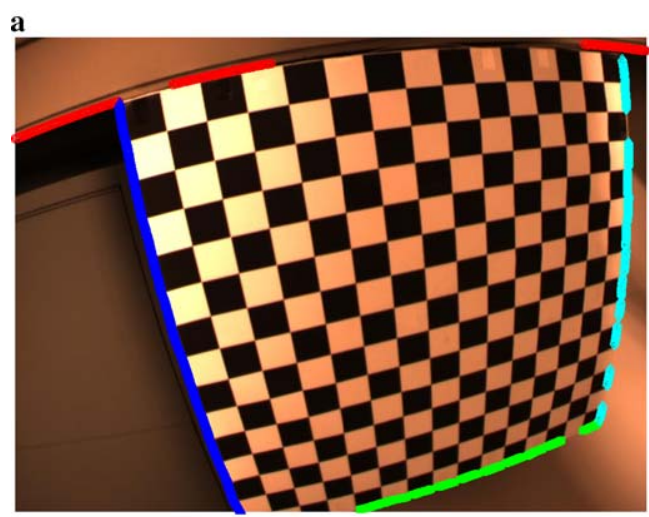

Original Image

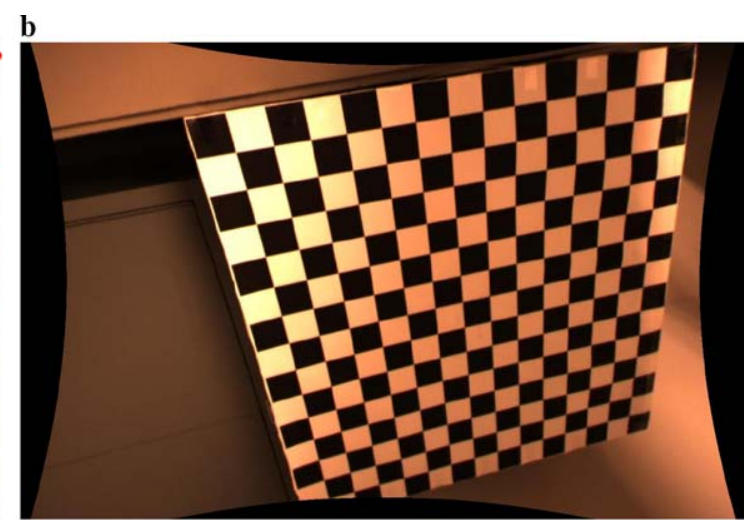

Corrected Image

Fig. 4. Estimation of radial distortion using line projections. On the left we show one of the six images used in this experiment. The size of the image is $768 \times 1024$. For each test image we fitted 4 circles to the projection of 4 lines and estimated the radial distortion parameter $\xi$ (Eq. (28)). The mean value for the radial distortion estimation was $\xi=-7.3125 \times 10^{-7}$. It corresponds to a displacement along the radial direction of 273.55 pixels at the image corner. The standard deviation of the estimated displacement was 9.925 pixels. The correction of radial distortion using the estimated value is exhibited on the right side. 


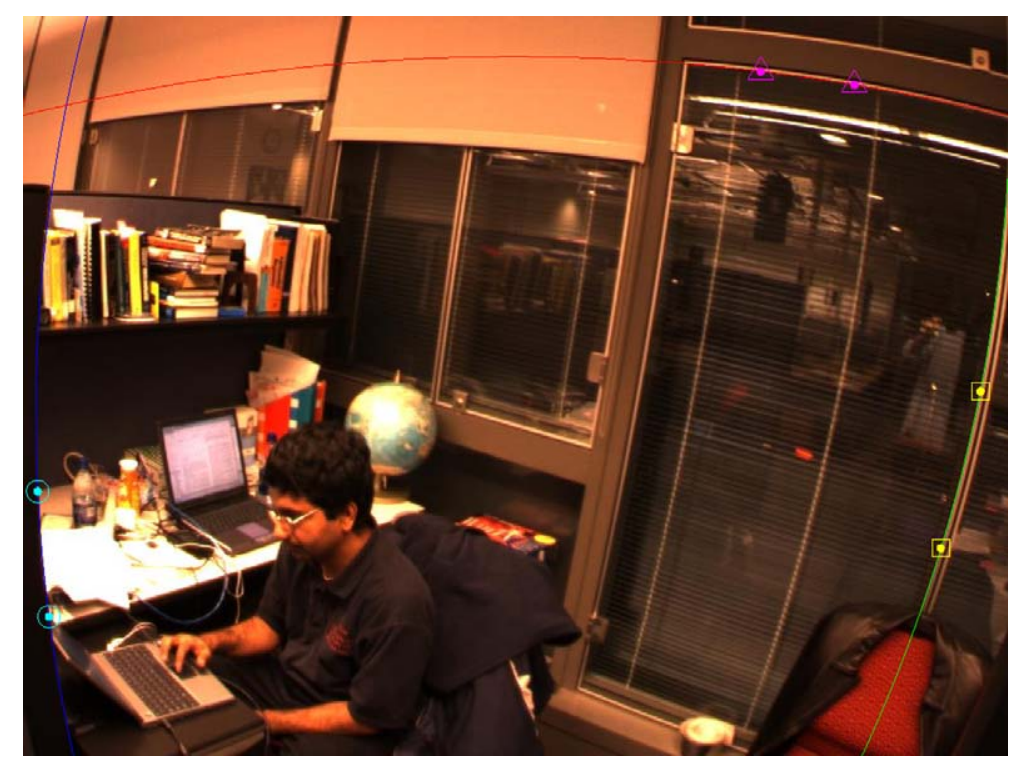

Fig. 5. Estimation of the distorted projection of a line from two image points selected by hand. The image size is $768 \times 1024$ and the radial distortion causes a displacement of 273.55 pixels at the image corner. The conic curve $\mathbf{\Omega}^{\prime \prime}$, corresponding to the projection of a line, is estimated from two image points knowing that $\boldsymbol{\Omega}^{\prime \prime}$ must go through the circular points and satisfies $\mathbf{M}^{\prime \prime} \boldsymbol{\Omega}^{\prime \prime} \mathbf{N}^{\prime \prime}=0$ (Eq. (27)).

As for the paracatadioptric camera situation, points $\omega^{\prime \prime}$ are the distorted projection of a line if, and only if, they lie in a certain plane of $\wp^{5 *}$. This plane is a subspace of the space of all circles, and the distortion can be easily estimated by fitting suitable circles to a set of line projections. The circles must follow the structure of Eq. (28) which implicitly encodes the distortion parameter $\xi$. Fig. 4a shows an image acquired by a camera with significant lens distortion. We applied and edge detector in order to measure image points $\mathbf{x}^{\prime \prime}$ lying on 4 distinct line projections. We fitted 4 consistent circles to the data points using normal least squares (Eq. (28)). The procedure was repeated for 5 other images acquired by the same camera. The mean estimation for the radial distortion was $\xi=-7.3125 \times 10^{-7}$.

In [22], we propose an algorithm to robustly estimate the projection of lines in calibrated paracatadioptric images. In the experiment of Fig. 5 we extend the method to the case of cameras with radial distortion. This extension is trivial given the observed similarities in the geometry of line projection. In Section 4.3, we saw that a conic curve $\boldsymbol{\Omega}^{\prime \prime}$ is the distorted projection of a line if, and only if, it goes through the circular points $\mathbf{I}^{\prime \prime}$ and $\mathbf{J}^{\prime \prime}$ and satisfies $\mathbf{M}^{\prime \prime} \mathbf{\Omega}^{\prime \prime} \mathbf{N}^{\prime \prime}=0$. Remark that points $\mathbf{M}^{\prime \prime}$ and $\mathbf{N}^{\prime \prime}$ only depend on the distortion parameter $\xi$ that is known (Eq. (27)). Since a conic curve has 5 degrees of freedom, the line projection $\boldsymbol{\Omega}^{\prime \prime}$ is fully constrained by knowing 2 image points lying on it. Fig. 5 shows the loci where three lines in the scene are projected. Each curve was determined using just two image points selected by hand.

\section{Conclusion}

In this paper, we studied unifying models for central projection systems and representations of projections of world points and lines. We first proved that the two step projection model through the sphere, equivalent to perspective cameras and all central catadioptric systems, can be extended to cover the division model of radial lens distortion. Having accommodated all central catadioptric as well as radial lens distortion models under one formulation, we established a representation of the resulting image planes in the five-dimensional projective space through the Veronese mapping. In this space, a collineation of the original plane corresponds to a collineation of the lifted space. Projections of lines in the world correspond to points in the lifted space lying in the general case on a quadric surface. However, in the cases of paracatadioptric and radial lens distortions, liftings of the projections of world lines lie on hyperplanes. In ongoing work, we study the epipolar geometry of central camera systems when points are expressed in this lifted space.

\section{Acknowledgments}

The author is grateful for support through the following Grants: NSF-IIS-0083209, NSF-IIS-0121293, NSF-EIA0324977, and ARO/MURI DAAD19-02-1-0383. Generous funding was also supplied by the Luso-American Foundation for Development.

\section{References}

[1] R. Hartley, A. Zisserman, Multiple View Geometry in Computer Vision, Cambridge University Press, Cambridge, MA, 2000.

[2] S. Baker, S. Nayar, A theory of catadioptric image formation, in: Proceedings of IEEE International Conference on Computer Vision, Bombay, 1998.

[3] T. Svoboda, T. Pajdla, Epipolar geometry for central catadioptric cameras, Int. J. Computer Vision 49 (1) (2002) 23-37. 
[4] C. Geyer, K. Danilidis, An unifying theory for central panoramic systems and pratical implications, in: Proceedings of European Conference on Computer Vision, Dublin, 2000, pp. $445-461$.

[5] D.C. Brown, Decentering distortion of lens, Photogramm. Eng. 32 (3) (1966) $444-462$.

[6] R. Willson, S. Shaffer, What is the center of the image, in: Proceedings of IEEE International Conference on Computer Vision and Pattern Recognition, 1993.

[7] C. Brauer-Burchardt, K. Voss, A new algorithm to correct fish-eye and strong wide angle lens distortion from single images, in Proceedings of IEEE International Conference on Image Processing, Thessaloniki, Greece, 2001.

[8] A. Fitzgibbon, Simultaneous linear estimation of multipleview geometry and lens distortion, in: Proceedings of IEEE International Conference on Computer Vision and Pattern Recognition, Haway, USA, 2001.

[9] J. Stolfi, Oriented Projective Geometry, Academic Press, New York, 1991.

[10] J.P. Barreto, H. Araujo, Geometric properties of central catadioptric line images and its application in calibration, IEEE Trans. Pattern Anal. Mach. Intell. 27 (8) (2005) 1327-1333.

[11] L. Wolf, A. Shashua, On projection matrices $\mathbf{P}^{\mathbf{k}} \rightarrow \mathbf{P}^{2}$, and their applications in computer vision, in: Proceedings of IEEE International Conference on Computer Vision, 2001.

[12] C. Geyer, K. Daniilids, Mirrors in motion. Epipolar geometry and motion estimation, in: Proceedings of ICCV'2003-IEEE International Conference on Computer Vision, Nice, France, 2003 .
[13] P. Sturm, Mixing catadioptric and perspective cameras, in: Proceedings of IEEE Workshop on Omnidirectional Vision, Copenhagen, Denmark, 2002.

[14] J.G. Sample, G.T. Kneebone, Algebraic Projective Geometry, Clarendon Press, Oxford, 1998.

[15] J.G. Sample, L. Roth, Algebraic Geometry, Clarendon Press, Oxford, 1949.

[16] D. Feldman, T. Padjla, D. Weinshall, On the epipolar geometry of the crossed-slits projection, in: Proceedings of ICCV'2003-IEEE International Conference on Computer Vision, Nice, France, 2003.

[17] R. Vidal, Y. Ma, S. Sastry, Generalized principal component analysis (gpca), in: Proceedings of CVPR'2003-IEEE International Conference on Computer Vision and Pattern Recognition, Madison, Wisconsin, 2003.

[18] F. Klein, Elementary Mathematics from an Advanced Standpoint, Macmillan, New York, 1939.

[19] X. Ying, Z. Hu, Catadioptric camera calibration using geometric invariants, IEEE Trans. Pattern Anal. Mach. Intell. 26 (10) (2004) $1260-1271$

[20] X. Ying, Z. Hu, Can we consider central catadioptric cameras and fisheye cameras within a unified imaging model?, in: Proceedings of ECCV'2004-European Conference in Computer Vision, Prague, Czech Republic, 2004.

[21] J. P. Barreto, H. Araujo, Paracatadioptric camera calibration using lines, in: Proceedings of ICCV'2003-IEEE International Conference on Computer Vision, Nice, France, 2003.

[22] J. P. Barreto, H. Araujo, Direct least square fitting of paracatadioptric line images, in: Proceedings of OMNIVIS'2003-Workshop on Omnidirectional Vision and Camera Networks, Madison, Wisconsin, 2003. 